\title{
Loss Parameter Calculations
}

\author{
J. M. Cook
}

\section{Introduction}

In reference [1] Sands and Rees propose an electronic bench measurement of the impulse energy loss of a stored particle bunch to vacuum-chamber components. The components act as the outer conductor of a coaxial line with a thin wire as center conductor. Short pulses are then transmitted through this coaxial system to simulate relativistic particle bunches. Their proposal has since been implemented by several investigators $[2,3,4,5,6]$ and has become a well-known technique.

They derive a first-order approximation to the loss parameter $k$ for use in these measurements. The purpose of this note is to point out that the exact expression for $k$ is as simple as its first-order approximation and to recommend its use even when $k$ is small.

\section{A First-Order Approximation}

In an equivalent circuit model of the system, the value $i(t)$ of the function $i$ represents the current that is at time $t$ entering the chamber being tested. To record $i$, the coaxial cable from the pulse generator is split as shown in Figure 2 of [6] and the reference chamber shown there in parallel with the test chamber is removed so that the sampling head of the corresponding oscilloscope is symmetric with the entrance to the test chamber. The impedance of the sampling head is equal to $Z_{0}$, the characteristic impedance of the cable, so when the pulse deposits an amount ${ }^{1}$

$$
U=Z_{0} \int_{-\infty}^{+\infty} i(t)^{2} d t
$$

of energy into that resistance, an equal amount of energy is also put into the test component by the other half of the split pulse.

A second oscilloscope with sampling head also of resistance $Z_{0}$ is positioned at the exit from the chamber. Thus the system is instrumented for a complete set of input-output measurements with input to the left end of the chamber and output from the right end, from and to coaxial cables with characteristic impedance $Z_{0}$. There is no provision for input from

\footnotetext{
${ }^{1}$ See equation (B-1) on page 38 of [1].
} 


\section{DISCLAIMER}

Portions of this document may be illegible in electronic image products. Images are produced from the best available original document. 
the right (usually unnecessary by symmetry) or for the measurement of reflections to the left, back towards the pulse generator.

The energy deposited in the sampling head at the exit from the test chamber is equal to $U$ minus the energy intercepted by the chamber. Subtract the input pulse from the output pulse to form $\delta i$. Then the first-order approximation to the energy change, ${ }^{2}$ as given by the variational calculus (see $\S 3.2$ in [7]), is the first variation of $U$ :

$$
\begin{aligned}
\delta U & =\delta\left(Z_{0} \int_{-\infty}^{+\infty} i(t)^{2} d t\right) \\
& =Z_{0} \int_{-\infty}^{+\infty} \delta\left(i(t)^{2}\right) d t \\
& =2 Z_{0} \int_{-\infty}^{+\infty} i(t) \cdot(\delta i)(t) d t .
\end{aligned}
$$

By definition of $k,{ }^{3}$

$$
-\Delta U=k q^{2}
$$

where $q=\int_{-\infty}^{+\infty} i(t) d t$, and $\Delta U$ is the exact increment of $U$ corresponding to the increment $\delta i$ of $i$. (Because it is the independent variable, $\Delta i=\delta i{ }^{4}$ ) Therefore ${ }^{5}$ the first approximation to $k$ is given by

$$
k \approx-\delta U / q^{2}=-2 Z_{0} \int_{-\infty}^{+\infty} i(t) \cdot \delta i(t) d t / q^{2} .
$$

\section{An Exact Expression}

The "exact" expression will be obtained by repeating arguments of Sands and Rees except where they explicitly state that they are neglecting terms of order higher than one. Then we simply keep all of those terms. The resulting expression will be exact in the sense that it follows from the arguments of Sands and Rees. If those arguments are not valid then their first-order approximation to $k$ should not be used without some other derivation.

Higher order approximations to $k$ can be obtained by adding to $\delta U$ in the above expression higher order terms in the Taylor expansion

$$
\Delta U=\delta U+\frac{\delta^{2} U}{2 !}+\frac{\delta^{3} U}{3 !}+\cdots
$$

(see page 87 of [8]). But $U$ is a quadratic functional so the variational derivatives $\delta^{n} U / \delta i^{n}$ are all zero for $n>2$ (see $\S 24$ in [7]) and the Taylor expansion reduces to

$$
\Delta U=2 Z_{0} \int_{-\infty}^{+\infty} i(t)(\delta i)(t) d t
$$

\footnotetext{
${ }^{2}$ See the first equality in equation B-6 on page 39 of [1].

${ }^{3}$ See equation (8) on page 16 of [1].

${ }^{4}$ In equations (B-3) and (B-4) on page 38 of [1], $\Delta i$ is denoted by $-i_{a}$. We expect it to be mostly negative (see line 4 on page 41 of [1]).

${ }^{5}$ See equation 10 on page 16 of [1]. The minus sign corresponds to the choice of the sign of $\delta i$.
} 


$$
\begin{aligned}
& +Z_{0} \int_{-\infty}^{+\infty}(\delta i(t))^{2} d t \\
& +0 .
\end{aligned}
$$

This is an algebraic identity easily verified by purely algebraic manipulations without use of any fancy derivatives. ${ }^{6}$ The exact formula for $k$ is then obtained from its first-order approximation simply by replacing $\delta U$ there by $\Delta U$ :

$$
\begin{aligned}
k & =-\Delta U / q^{2} \\
& =-Z_{0} \int_{-\infty}^{+\infty}(2 i(t)+\delta i(t)) \cdot \delta i(t) d t / q^{2}
\end{aligned}
$$

The inaccuracy in the first-order approximation is given by the second-order term, so the approximation is always larger than the exact value. In references $[1,2,3,4,5,6]$ the inaccuracy must be reduced (but not eliminated) by shifting the data describing the output pulse backwards to correct for the time-delay as the pulse traverses the chamber. Minimization of the second-order term is then just a one-parameter least-squares fit. As so determined, $k$ is an insensitive function of the shift because the minimum is characterized by a zero of the derivative of that function. However in [6], as in earlier papers, the shift was determined by bringing into coincidence the leading edges of the two pulses. The particular algorithm used in [6] was quite effective at obtaining this coincidence but, unlike the minimization algorithm which compares the entire pulse-shapes, it compares only the shapes of the leading edges. At the suggestion of Gil Nicholls the sensitivity of the first-order-approximate $k$ to this shift was investigated. It was found to be so high as to threaten the validity of the approximation. On the other hand, the value of the exact expression is invariant under time-translation. No shift is necessary.

Alternatively, $\Delta U$ can be evaluated directly from its definition:

$$
\Delta U=U(i+\delta i)-U(i)^{7}
$$

where $i$ is the input pulse.

In some of the experiments done for [6], two components to be compared were tested simultaneously, in parallel (see figure 2). For example the value of $k$ for a hardline as reference versus hardline-plus-pillbox for test component was computed in by the first-order

\footnotetext{
${ }^{6}$ Analogously, the higher order terms neglected by Sands and Rees in the first part of equation (B-6) on page 39 of [1] can be recovered in the derivation of $2 R_{0} i \Delta i \Delta t$ from $R_{0} i^{2} \Delta t$ two lines above by noticing that $\Delta i^{2}=(i+\Delta i)^{2}-i^{2} \equiv 2 \Delta i+(\Delta i)^{2}$, and then keeping the second-order term $(\Delta i)^{2}$.

${ }^{7}$ On page 38 of [1], energy dissipated in the chamber by the coaxial current pulse is neglected. Instead, the energy lost by the pulse is "...to a large extent coupled into the center wire ...to be dissipated in the terminations at the two ends" (page 7, lines 5-7). (On the other hand, the energy lost by a beam bunch "is transformed eventually into heat energy in the walls" (page 7, line 2). The central assumption of [1] is that both losses are equal ("...similar ...", page 7, lines 12-14).) Therefore on page 38, where only the coaxial current pulse is discussed, equations (B-2) through (B-4) imply $\Delta U=U(i+\delta i)-U(i)=$ the energy $R_{0} \int i_{b}^{2}(t) d t$ lost to the reflected pulse.

The authors then go on to describe something they would "like" in the line at the bottom of page 38 and the next three lines at the top of page 39 but I cannot find any supporting arguments for this preference.
} 
approximation to be .20703 volts/picocoulomb. When computed by the exact expression the value is $.12569 \mathrm{~V} / \mathrm{pC}$. The difference, .08134 , is the second-order correction term. This is a relatively large number, and the situation does not improve much when $k$ is small. For an elliptical beam chamber as reference versus beam tube plus abrupt transition to antechamber, typically the first-order approximation to $k$ was about $-.003 \mathrm{~V} / \mathrm{pC}$ whereas the exact value was about $-.004 \mathrm{~V} / \mathrm{pC}$, a significant difference.

\section{References}

[1] M. Sands and J. Rees, A Bench Measurement of the Energy Loss of a Stored Beam to a Cavity, PEP-95, Stanford Linear Accelerator Center, Palo Alto, California, August 8, 1974.

[2] J. Peters, Bench Measurements of the Energy Loss of a Stored Beam to Vacuum Components, IEEE NS-24, 1446, (1977).

[3] P. B. Wilson et al., Comparison of Measured and Computed Loss to Parasitic Modes in Cylindrical Cavities with Beam Ports, IEEE NS-24, 1496, (1977).

[4] M. J. Billing et al., Simulation Measurement of Bunch Excited Fields and Energy Loss in Vacuum Chamber Components and Cavities, IEEE NS-26, 3583, (1979).

[5] J. N. Weaver et al., Bench Measurements of Loss Impedance for PEP Beam Line Components, IEEE NS-26, 3971, (1979).

[6] R. Kustom et al., RF Impedance Studies of a Beam Chamber and Longitudinally Slot-Coupled Vacuum Pumping Antechamber, ANL-HEP-CP-87-19, published in IEEE Transactions on Nuclear Science, Conference Records 87CH2387-9, IEEE Particle Accelerator Conference, Accelerator Engineering and Technology, March 16-19, 1987, Washington, D.C., Volume 2, page 1304.

[7] I. M. Gelfand and S. V. Fomin, Calculus of Variations, Prentice-Hall, Inc., Englewood Cliffs, N. J. (1963).

[8] G. M. Ewing, Calculus of Variations with Applications, Dover Publications, Inc., New York (1985).

\section{DISCLAIMER}

This report was prepared as an account of work sponsored by an agency of the United States Government. Neither the United States Government nor any agency thereof, nor any of their employees, makes any warranty, express or implied, or assumes any legal liability or responsibility for the accuracy, completeness, or usefulness of any information, apiaaratus, product, or process disclosed, or represents that its use would not infringe privately owned rights. Reference herein to any specific commercial product, process, or service by trade name, trademark, manufacturer, or otherwise does not necessarily constitute or imply its endorsement, recommendation, or favoring by the United States Government or any agency thereof. The views and opinions of authors expressed herein do not necessarily state or reflect those of the United States Government or any agency thereof. 